\title{
Frank-Ter Haar syndrome
}

INSERM

\section{Source}

INSERM. (1999). Orphanet: an online rare disease and orphan drug data base. Frank-Ter Haar syndrome. ORPHA:137834

Frank-ter Haar syndrome (formerly considered as an autosomal recessive form of Melnick-Needles syndrome; see this term) is defined by megalocornea, multiple skeletal anomalies, characteristic facial dysmorphism (wide fontanels, prominent forehead, hypertelorism, prominent eyes, full cheeks and microg nathia) and developmental delay. 